\title{
Asymptotical Convergence of the Solutions of a Linear Differential Equation with Delays
}

\author{
Josef Diblík, Miroslava Růžičková, and Zuzana Šutá \\ Department of Mathematics, University of Žilina, Faculty of Science, Univerzitná 8215/1, \\ 01026 Žilina, Slovakia \\ Correspondence should be addressed to Josef Diblík, josef.diblik@fpv.uniza.sk
}

Received 1 January 2010; Accepted 23 April 2010

Academic Editor: Ağacik Zafer

Copyright (C) 2010 Josef Diblík et al. This is an open access article distributed under the Creative Commons Attribution License, which permits unrestricted use, distribution, and reproduction in any medium, provided the original work is properly cited.

The asymptotic behavior of the solutions of the first-order differential equation $\dot{y}(t)=$ $\sum_{i=1}^{n} \beta_{i}(t)\left[y\left(t-\delta_{i}\right)-y\left(t-\tau_{i}\right)\right]$ containing delays is studied with $\beta_{i}:\left[t_{0}-\tau, \infty\right) \rightarrow[0, \infty)$, $\tau=\max \left\{\tau_{1}, \ldots, \tau_{n}\right\}, \sum_{i=1}^{n} \beta_{i}(t)>0, \tau_{i}>\delta_{i}>0$. The attention is focused on an analysis of the asymptotical convergence of solutions. A criterion for the asymptotical convergence of all solutions, characterized by the existence of a strictly increasing bounded solution, is proved. Relationships with the previous results are discussed, too.

\section{Introduction}

We investigate the asymptotic behavior of the solutions of a linear homogeneous differential equation with delayed terms

$$
\dot{y}(t)=\sum_{i=1}^{n} \beta_{i}(t)\left[y\left(t-\delta_{i}\right)-y\left(t-\tau_{i}\right)\right]
$$

as $t \rightarrow \infty$. In (1.1) we assume $\delta_{i}, \tau_{i} \in \mathbb{R}^{+}=(0,+\infty), \tau_{i}>\delta_{i}$, functions $\beta_{i}: I_{-1} \rightarrow \mathbb{R}_{+}:=[0,+\infty)$ where $I_{-1}:=\left[t_{0}-\tau, \infty\right), t_{0} \in \mathbb{R}, \tau=\max \left\{\tau_{1}, \ldots, \tau_{n}\right\}$ are continuous and such that $\sum_{i=1}^{n} \beta_{i}(t)>0$ on $I_{-1}$. Set $I:=\left[t_{0}, \infty\right)$. Throughout the paper the symbol "." denotes the right-hand derivative. Similarly, if necessary, the value of a function at a point of $I_{-1}$ is understood as the value of the corresponding limit from the right.

We call a solution $y=y(t)$ of (1.1) asymptotically convergent if it has a finite limit $\lim _{t \rightarrow \infty} y(t)$. The main results concern the asymptotical convergence of all solutions of (1.1). Besides, the proof of the results is based on the comparison of solutions of (1.1) with solutions 
of an auxiliary inequality which formally copies (1.1). At first, we prove that, under certain conditions, (1.1) has a strictly increasing asymptotically convergent solution. Then we extend this statement to all the solutions of (1.1). Moreover, in the general case, the asymptotical convergence of all solutions is characterized by the existence of a strictly increasing bounded solution.

The problem concerning the asymptotical convergence of solutions of delayed differential equations (or delayed difference equation, etc.) is a classical one. But the problem of the asymptotic convergence or divergence of solutions of delayed equations receives permanent attention. Let us mention at least investigations [1-18]. Comparing the known investigations with the results presented we conclude that our results give more sharp sufficient conditions.

The paper is organized as follows. In Section 2 an auxiliary inequality is studied and the relationship of its solutions with solutions of (1.1) is derived. The existence of a strictly increasing and convergent solution of (1.1) is established in Section 3. Section 4 contains results concerning the asymptotical convergence of all the solutions of (1.1). The related previous results are discussed in Section 5.

Let $\mathcal{C}:=C([-\tau, 0], \mathbb{R})$ be the Banach space of continuous functions mapping the interval $[-\tau, 0]$ into $\mathbb{R}$ equipped with the supremum norm.

Let $v \in I$ be given. The function $y:[v-\tau, \infty) \rightarrow \mathbb{R}$ is said to be a solution of (1.1) on $[v-\tau, \infty)$ if $y$ is continuous on $[v-\tau, \infty)$, continuously differentiable on $[v, \infty)$ and satisfies (1.1) for $t \in[v, \infty)$.

For $v \in I, \varphi \in \mathcal{C}$, we say that $y(v, \varphi)$ is a solution of $(1.1)$ through $(v, \varphi)$ (or that $y(v, \varphi)$ corresponds to the initial point $v)$ if $y(v, \varphi)$ is a solution of $(1.1)$ on $[v-\tau, \infty)$ and $y(v, \varphi)(v+\theta)=$ $\varphi(\theta)$ for $\theta \in[-\tau, 0]$.

\section{Auxiliary Inequality}

The inequality

$$
\dot{\omega}(t) \geq \sum_{i=1}^{n} \beta_{i}(t)\left[\omega\left(t-\delta_{i}\right)-\omega\left(t-\tau_{i}\right)\right]
$$

plays an important role in the analysis of (1.1). Let $v \in I$ and $A>0$ be given. The function $\omega:[v-\tau, v+A) \rightarrow \mathbb{R}$ is said to be a solution of (2.1) on $[v-\tau, v+A)$ if $\omega$ is continuous on $[v-\tau, v+A)$, continuously differentiable on $[v, v+A)$, and satisfies inequality (2.1) for $t \in[v, v+A)$. If $A=\infty$, we call the solution $\omega$ of (2.1) asymptotically convergent if it has a

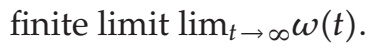

\subsection{Relationship between the Solutions of Inequality (2.1) and Equation (1.1)}

In this part, we will derive some properties of the solutions of type (2.1) inequalities and compare the solutions of (1.1) with those of inequality (2.1).

Lemma 2.1. Let $\varphi \in \mathcal{C}$ be strictly increasing (nondecreasing, strictly decreasing, nonincreasing) on $[-\tau, 0]$. Then the corresponding solution $y\left(t^{*}, \varphi\right)(t)$ of $(1.1)$ with $t^{*} \in I$ is strictly increasing (nondecreasing, strictly decreasing, nonincreasing) on $\left[t^{*}-\tau, \infty\right)$, respectively. If $\varphi$ is strictly 
increasing (nondecreasing) and $\omega: I_{-1} \rightarrow R$ is a solution of $(2.1)$ with $\omega\left(t_{0}+\theta\right)=\varphi(\theta), \theta \in[-1,0]$, then $\omega$ is strictly increasing (nondecreasing) on $I_{-1}$.

Proof. This is clear from (1.1) and (2.1) and from $\beta_{i}(t) \geq 0, \sum_{i=1}^{n} \beta_{i}(t)>0, t \in I, \tau_{i}>\delta_{i}>0$, $i=1,2, \ldots, n$.

Theorem 2.2. Let $\omega(t)$ be a solution of inequality (2.1) on $I_{-1}$. Then there exists a solution $y(t)$ of (1.1) on $I_{-1}$ such that the inequality

$$
y(t) \leq \omega(t)
$$

holds on $I_{-1}$. In particular, a solution $y\left(t_{0}, \phi\right)$ of $(1.1)$ with $\phi \in \mathcal{C}$ defined by the relation

$$
\phi(\theta):=\omega\left(t_{0}+\theta\right), \quad \theta \in[-\tau, 0]
$$

is such a solution.

Proof. Let $\omega(t)$ be a solution of inequality (2.1) on $I_{-1}$. We will show that the solution $y(t):=$ $y\left(t_{0}, \phi\right)(t)$ of $(1.1)$ satisfies inequality (2.2), that is,

$$
y\left(t_{0}, \phi\right)(t) \leq \omega(t)
$$

on $I_{-1}$. Define on $I_{-1}$ the continuous function $W(t)=\omega(t)-y(t)$. Then $W=0$ on $\left[t_{0}-\tau, t_{0}\right]$, and $W$ is a solution of (2.1) on $I_{-1}$. Lemma 2.1 implies that $W$ is nondecreasing. Consequently, $\omega(t)-y(t) \geq \omega\left(t_{0}\right)-y\left(t_{0}\right)=0$ for all $t \geq t_{0}$.

Remark 2.3. Let us note that the assertion, opposite in a sense with to that statement of Theorem 2.2, is obvious. Namely, if a solution $y(t)$ of $(1.1)$ on $I_{-1}$ is given, then there exists a solution $\omega(t)$ of inequality (2.1) on $I_{-1}$ such that the inequality

$$
\omega(t) \leq y(t)
$$

holds on $I_{-1}$ since it can be put $\omega(t) \equiv y(t)$. Moreover, if we put, for example, $\omega_{1}(t) \equiv y(t)-1$, then

$$
\omega_{1}(t)<y(t)
$$

on $I_{-1}$.

\subsection{A Solution of Inequality (2.1)}

It is easy to get a solution of inequality (2.1) in an exponential form. We will indicate this form in the following lemma. This auxiliary result will help us derive concrete sufficient conditions for the existence of strictly increasing and convergent solution of (1.1). 
Lemma 2.4. Let there exist a function $\varepsilon: I_{-1} \rightarrow \mathbb{R}$, continuous on $I_{-1} \backslash\left\{t_{0}\right\}$ with at most first-order discontinuity at the point $t=t_{0}$ and satisfying the inequality

$$
\varepsilon(t)+\sum_{i=1}^{n} \beta_{i}(t) \exp \left[-\int_{t-\tau_{i}}^{t} \varepsilon(s) \mathrm{d} s\right] \geq \sum_{i=1}^{n} \beta_{i}(t) \exp \left[-\int_{t-\delta_{i}}^{t} \varepsilon(s) \mathrm{d} s\right]
$$

on $I$. Then there exists a solution $\omega(t)=\omega_{\mathrm{e}}(t)$ of inequality (2.1), defined on $I_{-1}$, and having the form

$$
\omega_{\mathrm{e}}(t):=\exp \left[\int_{t_{0}-\tau}^{t} \varepsilon(s) \mathrm{d} s\right] .
$$

Proof. Inequality (2.7) follows immediately from inequality (2.1) for $\omega=\omega_{\mathrm{e}}$.

\section{Existence of an Asymptotically Convergent Solution of (1.1)}

In this part we indicate sufficient conditions for the existence of a convergent solution of (1.1). First, let us introduce two obvious statements concerning asymptotical convergence. From Theorem 2.2 and Lemma 2.1, we immediately get the following.

Theorem 3.1. If $\omega$ is a strictly increasing asymptotically convergent solution of (2.1) on $I_{-1}$, then there exists a strictly increasing asymptotically convergent solution $y(t)$ of (1.1) on $I_{-1}$.

From Lemma 2.1, Theorem 2.2, and Lemma 2.4, we get the following.

Theorem 3.2. If there exists a function $\varepsilon: I_{-1} \rightarrow \mathbb{R}^{+}$, continuous on $I_{-1} \backslash\left\{t_{0}\right\}$ with at most the first order discontinuity at the point $t=t_{0}$ satisfying $\int^{\infty} \varepsilon(s) \mathrm{d} s<\infty$, and the inequality (2.7) on I, the initial function

$$
\varphi(\theta)=\exp \left[\int_{t_{0}-\tau}^{t_{0}+\theta} \varepsilon(s) \mathrm{d} s\right], \quad \theta \in[-\tau, 0]
$$

defines a strictly increasing and asymptotically convergent solution $y\left(t_{0}, \varphi\right)(t)$ of $(1.1)$ on $I_{-1}$ satisfying the inequality

$$
y(t) \leq \exp \left[\int_{t_{0}-\tau}^{t} \varepsilon(s) \mathrm{d} s\right]
$$

on I.

Theorem 3.3. If there exists a constant $\alpha>1$ such that

$$
\liminf _{t \rightarrow \infty} t \cdot\left[1+\sum_{i=1}^{n} \beta_{i}(t)\left[\delta_{i}-\tau_{i}-\frac{\alpha}{2 t}\left(\tau_{i}^{2}-\delta_{i}^{2}\right)\right]\right]>0,
$$

there exists a strictly increasing and asymptotically convergent solution $y(t)$ of (1.1) as $t \rightarrow \infty$. 
Proof. Let us verify that the integral inequality (2.7) has (for every sufficiently large $t$ ) a solution $\varepsilon$ such that

$$
\int^{\infty} \varepsilon(s) \mathrm{d} s<\infty
$$

In inequality (2.7), we put

$$
\varepsilon(t):=t^{-\alpha}
$$

where $\alpha>1$. Then (3.4) holds. Now we perform an auxiliary asymptotical analysis for $t \rightarrow \infty$. The symbol $O$ used below is the Landau order symbol. All asymptotical decompositions are developed with sufficient accuracy. Let $\gamma$ be a nonzero constant. Then

$$
\begin{aligned}
\int_{t-\gamma}^{t} \varepsilon(s) \mathrm{d} s & =\int_{t-\gamma}^{t} \frac{1}{s^{\alpha}} \mathrm{d} s \\
& =\frac{1}{1-\alpha}\left[\frac{1}{t^{\alpha-1}}-\frac{1}{(t-\gamma)^{\alpha-1}}\right] \\
& =\frac{1}{1-\alpha}\left[\frac{1}{t^{\alpha-1}}-\frac{1}{t^{\alpha-1}}\left(1-\frac{\gamma}{t}\right)^{1-\alpha}\right] \\
& =\frac{1}{1-\alpha}\left[\frac{1}{t^{\alpha-1}}-\frac{1}{t^{\alpha-1}}\left(1-\frac{(1-\alpha) \gamma}{t}-\frac{(1-\alpha) \alpha \gamma^{2}}{2 t^{2}}+O\left(\frac{1}{t^{3}}\right)\right)\right] \\
& =\frac{1}{t^{\alpha}}\left[\gamma+\frac{\alpha \gamma^{2}}{2 t}+O\left(\frac{1}{t^{2}}\right)\right]
\end{aligned}
$$

Moreover

$$
\begin{aligned}
\exp \left[-\int_{t-\gamma}^{t} \varepsilon(s) \mathrm{d} s\right] & =\exp \left[-\frac{1}{t^{\alpha}}\left[\gamma+\frac{\alpha \gamma^{2}}{2 t}+O\left(\frac{1}{t^{2}}\right)\right]\right] \\
& =1-\frac{1}{t^{\alpha}}\left[\gamma+\frac{\alpha \gamma^{2}}{2 t}+O\left(\frac{1}{t^{2}}\right)\right]+\frac{1}{2 t^{2 \alpha}}\left[\gamma+\frac{\alpha \gamma^{2}}{2 t}+O\left(\frac{1}{t^{2}}\right)\right]^{2}+O\left(\frac{1}{t^{3 \alpha}}\right) \\
& =1-\frac{\gamma}{t^{\alpha}}-\frac{\alpha \gamma^{2}}{2 t^{\alpha+1}}+O\left(\frac{1}{t^{\alpha+2}}\right)+O\left(\frac{1}{t^{2 \alpha}}\right)
\end{aligned}
$$

We use the asymptotical decomposition (3.7) and rewrite the integral inequality (2.7). We get

$$
\begin{aligned}
& \frac{1}{t^{\alpha}}+\sum_{i=1}^{n} \beta_{i}(t) {\left[1-\frac{\tau_{i}}{t^{\alpha}}-\frac{\alpha \tau_{i}^{2}}{2 t^{\alpha+1}}+O\left(\frac{1}{t^{\alpha+2}}\right)+O\left(\frac{1}{t^{2 \alpha}}\right)\right] } \\
& \geq \sum_{i=1}^{n} \beta_{i}(t)\left[1-\frac{\delta_{i}}{t^{\alpha}}-\frac{\alpha \delta_{i}^{2}}{2 t^{\alpha+1}}+O\left(\frac{1}{t^{\alpha+2}}\right)+O\left(\frac{1}{t^{2 \alpha}}\right)\right]
\end{aligned}
$$


or

$$
\frac{1}{t^{\alpha}}+\sum_{i=1}^{n} \beta_{i}(t)\left[-\frac{\tau_{i}-\delta_{i}}{t^{\alpha}}-\frac{\alpha\left(\tau_{i}^{2}-\delta_{i}^{2}\right)}{2 t^{\alpha+1}}+O\left(\frac{1}{t^{\alpha+2}}\right)+O\left(\frac{1}{t^{2 \alpha}}\right)\right] \geq 0
$$

Multiplying this inequality by $t^{\alpha+1}$, we get

$$
t\left[1+\sum_{i=1}^{n} \beta_{i}(t)\left[\delta_{i}-\tau_{i}-\frac{\alpha}{2 t}\left(\tau_{i}^{2}-\delta_{i}^{2}\right)+O\left(\frac{1}{t^{2}}\right)+O\left(\frac{1}{t^{\alpha}}\right)\right]\right] \geq 0
$$

Analysing inequality (3.10), we conclude that the inequality

$$
1+\sum_{i=1}^{n} \beta_{i}(t)\left(\delta_{i}-\tau_{i}\right)>0
$$

or, equivalently,

$$
\sum_{i=1}^{n} \beta_{i}(t)\left(\tau_{i}-\delta_{i}\right)<1
$$

is a necessary condition for its validity as $t \rightarrow \infty$ (because $\alpha>1$ and $\left.\tau_{i}>\delta_{i}, i=1, \ldots, n\right)$. Consequently,

$$
\beta_{i}(t)<\frac{1}{\tau_{i}-\delta_{i}} \leq M:=\max _{i=1, \ldots, n}\left\{\frac{1}{\tau_{i}-\delta_{i}}\right\}, \quad i=1, \ldots, n, t \in I
$$

Then, inequality (3.10) will be valid as $t \rightarrow \infty$ if there exist positive constants $d$ and $\pi \in$ $(1, \min \{2, \alpha\})$ such that

$$
\begin{aligned}
t\left[1+\sum_{i=1}^{n} \beta_{i}(t)\left[\delta_{i}-\tau_{i}-\frac{\alpha}{2 t}\left(\tau_{i}^{2}-\delta_{i}^{2}\right)+O\left(\frac{1}{t^{2}}\right)+O\left(\frac{1}{t^{\alpha}}\right)\right]\right] \\
>t\left[1+\sum_{i=1}^{n} \beta_{i}(t)\left[\delta_{i}-\tau_{i}-\frac{\alpha}{2 t}\left(\tau_{i}^{2}-\delta_{i}^{2}\right)\right]-\frac{d n M}{t^{\pi}}\right] \\
=t\left[1+\sum_{i=1}^{n} \beta_{i}(t)\left[\delta_{i}-\tau_{i}-\frac{\alpha}{2 t}\left(\tau_{i}^{2}-\delta_{i}^{2}\right)\right]\right]-\frac{d n M}{t^{\pi-1}}>0 .
\end{aligned}
$$

Inequality (3.3) implies that there is a positive constant $d^{*}$ such that

$$
t\left[1+\sum_{i=1}^{n} \beta_{i}(t)\left[\delta_{i}-\tau_{i}-\frac{\alpha}{2 t}\left(\tau_{i}^{2}-\delta_{i}^{2}\right)\right]\right]>d^{*}
$$


Finally, since

$$
t\left[1+\sum_{i=1}^{n} \beta_{i}(t)\left[\delta_{i}-\tau_{i}-\frac{\alpha}{2 t}\left(\tau_{i}^{2}-\delta_{i}^{2}\right)\right]\right]-\frac{d n M}{t^{\pi-1}}>d^{*}-\frac{d n M}{t^{\pi-1}}>0
$$

as $t \rightarrow \infty$, we conclude that (3.14) holds and, consequently, the integral inequality (2.7) has a solution $\varepsilon(t)=t^{-\alpha}$ for every sufficiently large $t$. Lemma 2.4 holds. We finalize the proof by noticing that the statement of the theorem directly follows from Theorem 3.1.

Assuming that functions $\beta_{i}, i=1,2, \ldots, n, t \in I$ can be estimated by suitable functions, we will prove that (1.1) has an asymptotically convergent solution. This yields two interesting corollaries directly following from inequality (3.3) in Theorem 3.3.

Corollary 3.4. Let

$$
0 \leq \beta_{i}(t) \leq b_{i}-\frac{c_{i}}{t}
$$

where $b_{i}$ and $c_{i}$ are nonnegative constants and $\sum_{i=1}^{n} \beta_{i}(t)>0$ on I. If, moreover,

$$
\sum_{i=1}^{n} b_{i}\left(\tau_{i}-\delta_{i}\right)=q<1
$$

then there exists a strictly increasing and convergent solution $y(t)$ of (1.1) as $t \rightarrow \infty$.

Proof. We show that the inequality (3.3) in Theorem 3.3 holds for any $\alpha>1$. Estimating the left-hand side of (3.3), we get

$$
\begin{aligned}
\liminf _{t \rightarrow \infty} t \cdot\left[1+\sum_{i=1}^{n} \beta_{i}(t)\left[\delta_{i}-\tau_{i}-\frac{\alpha}{2 t}\left(\tau_{i}^{2}-\delta_{i}^{2}\right)\right]\right] \\
\geq \liminf _{t \rightarrow \infty} t \cdot\left[1-\sum_{i=1}^{n}\left(b_{i}-\frac{c_{i}}{t}\right)\left[\tau_{i}-\delta_{i}+\frac{\alpha}{2 t}\left(\tau_{i}^{2}-\delta_{i}^{2}\right)\right]\right] \\
=\liminf _{t \rightarrow \infty} t \cdot\left[(1-q)+\sum_{i=1}^{n} \frac{c_{i}}{t}\left(\tau_{i}-\delta_{i}\right)-\frac{\alpha}{2 t} \sum_{i=1}^{n}\left(b_{i}-\frac{c_{i}}{t}\right)\left(\tau_{i}^{2}-\delta_{i}^{2}\right)\right]>0
\end{aligned}
$$

since $q \in(0,1)$.

Corollary 3.5. Let

$$
0 \leq \beta_{i}(t) \leq b_{i}-\frac{c_{i}}{t}
$$


where $b_{i}$ and $c_{i}$ are nonnegative constants and $\sum_{i=1}^{n} \beta_{i}(t)>0$ on I. If, moreover,

$$
\sum_{i=1}^{n} b_{i}\left(\tau_{i}-\delta_{i}\right)=1
$$

and there exists a constant $\alpha>1$ such that

$$
\sum_{i=1}^{n} c_{i}\left(\tau_{i}-\delta_{i}\right)>\frac{\alpha}{2} \sum_{i=1}^{n} b_{i}\left(\tau_{i}^{2}-\delta_{i}^{2}\right)
$$

then there exists a strictly increasing and convergent solution $y=y(t)$ of (1.1) as $t \rightarrow \infty$.

Proof. Employing a part of the proof of Corollary 3.4 with $q=1$, we use inequality (3.22). Then

$$
\begin{aligned}
\liminf _{t \rightarrow \infty} t \cdot\left[1+\sum_{i=1}^{n} \beta_{i}(t)\left[\delta_{i}-\tau_{i}-\frac{\alpha}{2 t}\left(\tau_{i}^{2}-\delta_{i}^{2}\right)\right]\right] \\
\geq \liminf _{t \rightarrow \infty}\left[\sum_{i=1}^{n} c_{i}\left(\tau_{i}-\delta_{i}\right)-\frac{\alpha}{2} \sum_{i=1}^{n}\left(b_{i}-\frac{c_{i}}{t}\right)\left(\tau_{i}^{2}-\delta_{i}^{2}\right)\right] \\
=\sum_{i=1}^{n} c_{i}\left(\tau_{i}-\delta_{i}\right)-\frac{\alpha}{2} \sum_{i=1}^{n} b_{i}\left(\tau_{i}^{2}-\delta_{i}^{2}\right)>0 .
\end{aligned}
$$

Thus, the inequality (3.3) in Theorem 3.3 holds.

\section{Asymptotical Convergence of All Solutions}

In this part we prove results concerning the asymptotical convergence of all the solutions of (1.1). First, we use inequality (3.3) to establish conditions for the asymptotical convergence of all the solutions.

Theorem 4.1. Let there exist $\alpha>1$ such that inequality (3.3) holds. Then all the solutions of (1.1) are asymptotically convergent for $t \rightarrow \infty$.

Proof. First we prove that every solution defined by a monotone initial function is asymptotically convergent. We will assume that a monotone initial function $\varphi \in \mathcal{C}$ is given. For the definiteness, let $\varphi$ be strictly increasing or nondecreasing (the strictly decreasing or nonincreasing case can be dealt with in much the same way). By Lemma 2.1, the solution $y\left(t_{0}, \varphi\right)(t)$ is monotone (either strictly increasing or nondecreasing) on $I_{-1}$. In what follows, we will prove that $y\left(t_{0}, \varphi\right)$ is asymptotically convergent.

By Theorem 3.3, there exists a strictly increasing and asymptotically convergent solution $Y$ of (1.1) on $I_{-1}$. Without loss of generality, we can assume that $y\left(t_{0}, \varphi\right)(t) \not \equiv Y(t)$ on $I_{-1}$ since, in the opposite case, we can choose another initial function. Moreover, we can assume that both solutions $y\left(t_{0}, \varphi\right)(t)$ and $Y(t)$ are continuously differentiable on $I_{-1}$. In the opposite case, we can start our reasoning with the interval $I$ instead of $I_{-1}$, that is, we can 
replace $t_{0}-\tau$ by $t_{0}$ and $t_{0}$ by $t_{0}+\tau$. Similarly, without loss of generality we can assume that $\dot{Y}(t)>0, t \in\left[t_{0}-\tau, t_{0}\right]$. Hence, there is a $k>0$ such that

$$
\left.\left(\dot{Y}(t)-k \dot{y}\left(t_{0}, \varphi\right)(t)\right)\right|_{t \in\left[t_{0}-\tau, t_{0}\right]}=\left.(\dot{Y}(t)-k \dot{\varphi}(t))\right|_{t \in\left[t_{0}-\tau, t_{0}\right]}>0 .
$$

Then, by Lemma 2.1, $Y(t)-k y\left(t_{0}, \varphi\right)(t)$ is strictly increasing in $I_{-1}$. Thus

$$
\begin{gathered}
Y(t)-k y\left(t_{0}, \varphi\right)(t)>Y\left(t_{0}-\tau\right)-k y\left(t_{0}, \varphi\right)\left(t_{0}-\tau\right), \quad t>t_{0}-\tau, \\
y\left(t_{0}, \varphi\right)(t)<\frac{1}{k}\left(Y(t)-Y\left(t_{0}-\tau\right)\right)+y\left(t_{0}, \varphi\right)\left(t_{0}-\tau\right)
\end{gathered}
$$

is a bounded function for all $t>t_{0}-\tau$.

Summarizing the previous part, we state that every monotone solution is asymptotically convergent. It remains to consider a class of all nonmonotone initial functions. For the behavior of a solution $y\left(t_{0}, \varphi\right)$, generated by a nonmonotone initial function $\varphi \in \mathcal{C}$ there are two possibilities: either $y\left(t_{0}, \varphi\right)$ is eventually monotone and, consequently, asymptotically convergent, or $y\left(t_{0}, \varphi\right)$ is eventually nonmonotone.

We will also use the known fact that every absolutely continuous function can be decomposed into the difference of two strictly increasing absolutely continuous functions [19, page 315]. Assuming that an initial (nonmonotone) function $\varphi \in \mathcal{C}$ is absolutely continuous on interval $[-\tau, 0]$, we can decompose it on the interval $[-\tau, 0]$ into the difference $\varphi=\varphi_{1}-\varphi_{2}$ of two strictly increasing absolutely continuous functions $\varphi_{1} \in \mathcal{C}, \varphi_{2} \in \mathcal{C}$. In accordance with the previous part of the proof, every function $\varphi_{i}, i=1,2$ defines a strictly increasing convergent solution $y\left(t_{0}, \varphi_{i}\right)$. Now it becomes clear that the solution $y\left(t_{0}, \varphi\right)$ is asymptotically convergent. To complete the proof, it remains to prove that, without loss of generality, we can restrict the set of all initial functions to the set of absolutely continuous initial functions. To this end, we again consider the solution $y\left(t_{0}, \varphi\right)$ defined by $\varphi \in \mathcal{C}$ and, if necessary, always without loss of generality, we can replace $t_{0}-\tau$ by $t_{0}$ and $t_{0}$ by $t_{0}+\tau$ since the solution has a finite derivative the interval $\left[t_{0}, t_{0}+\tau\right]$. Finally, we remark that any function satisfying the Lipschitz condition on an interval $[a, b]$ is absolutely continuous in it [19, page 313].

Tracing the proof of Theorem 4.1, we can see that the inequality (3.3) was used only as "input" information stating that, in accordance with Theorem 3.3, there exists a strictly increasing and asymptotically convergent solution $Y$ of (1.1) on $I_{-1}$. If the existence of a strictly monotone and asymptotically convergent solution is assumed instead of (3.3), we obtain the following

Theorem 4.2. If (1.1) has a strictly monotone and convergent solution on $I_{-1}$, then all the solutions of (1.1) defined on $I_{-1}$ are asymptotically convergent.

Moreover, combining the results formulated in Theorems 2.2, 3.1 and 4.2, we obtain the following

Theorem 4.3. The following three statements are equivalent.

(a) Equation (1.1) has a strictly monotone and asymptotically convergent solution on $I_{-1}$.

(b) All solutions of (1.1) defined on $I_{-1}$ are asymptotically convergent.

(c) Inequality (2.1) has a strictly monotone and asymptotically convergent solution on $I_{-1}$. 


\section{Comparison with Previous Results}

In [8], conditions for the asymptotical convergence of all the solutions of (1.1) with $n=1$ and $\delta=0$, that is,

$$
\dot{y}(t)=\beta(t)[y(t)-y(t-\tau)]
$$

are given. A particular case of (1.1) with $n=1$, that is, the case

$$
\dot{y}(t)=\beta(t)[y(t-\delta)-y(t-\tau)]
$$

is treated, for example, in $[2,4,10,11]$. The following theorem (see [11, Theorems 4, 5, and 9]) gives corresponding results related to (5.2). Its first part gives sufficient conditions for the existence of a strictly increasing and unbounded solution of (5.2) whereas the second part provides sufficient conditions for the asymptotical convergence of all its solutions.

Theorem 5.1. (a) Let there exist a constant $p \in(0,1)$ such that the inequality

$$
\beta(t) \geq \frac{1}{\tau-\delta}-\frac{p}{2 t}
$$

holds for all $t \in I_{-1}$. Then there exists a strictly increasing and unbounded solution of (5.2) as $t \rightarrow \infty$.

(b) Let there exist a constant $p>1$ such that the inequality

$$
\beta(t) \leq \frac{1}{\tau-\delta}-\frac{p(\tau+\delta)}{2(\tau-\delta) t}
$$

holds for all $t \in I_{-1}$. Then all solutions of (5.2) defined on $I_{-1}$ are convergent.

Comparing (5.3) with (5.4), we see that the maximal allowed values for $\beta$ given in (5.4) are not strictly opposite with respect to the minimal allowed values for $\beta$ given in (5.3). This is a gap since

$$
\frac{\tau+\sigma}{\tau-\sigma}>1
$$

and one would expect a pair of opposite inequalities stronger than (5.3) and (5.4). Can one of the inequalities (5.3) or (5.4) be improved? The following example shows that probably (5.3) can be improved because (5.4) provides a best possible criterion.

Example 5.2. Consider (5.2) with $\beta(t)=[t \ln ((t-\delta) /(t-\tau))]^{-1}$, that is,

$$
\dot{y}(t)=\frac{1}{t \ln ((t-\delta) /(t-\tau))} \cdot(y(t-\delta)-y(t-\tau)) .
$$


It is easy to verify that $y(t)=\ln t$ is a strictly increasing and unbounded solution of (5.6) as $t \rightarrow \infty$. Decomposing asymptotically $\beta(t)$ as $t \rightarrow \infty$ we get

$$
\begin{aligned}
\beta(t) & =\frac{1}{t \ln ((t-\delta) /(t-\tau))}=\frac{1}{t[\ln (1-\delta / t)-\ln (1-\tau / t)]} \\
& =\frac{1}{t} \cdot \frac{1}{-\delta / t-\delta^{2} / 2 t^{2}-\delta^{3} / 3 t^{3}+\tau / t+\tau^{2} / 2 t^{2}+\tau^{3} / 3 t^{3}+O\left(1 / t^{4}\right)} \\
& =\frac{1}{\tau-\delta} \cdot\left[1+\frac{1}{2 t}(\tau+\delta)+\frac{1}{3 t^{2}}\left(\tau^{2}+\tau \delta+\delta^{2}\right)+O\left(\frac{1}{t^{3}}\right)\right]^{-1} \\
& =\frac{1}{\tau-\delta} \cdot\left[1-\frac{1}{2 t}(\tau+\delta)-\frac{1}{3 t^{2}}\left(\tau^{2}+\tau \delta+\delta^{2}\right)+\frac{1}{4 t^{2}}(\tau+\delta)^{2}+O\left(\frac{1}{t^{3}}\right)\right] \\
& =\frac{1}{\tau-\delta}-\frac{1}{2 t} \frac{\tau+\delta}{\tau-\delta}-\frac{\tau-\delta}{12 t^{2}}+O\left(\frac{1}{t^{3}}\right) .
\end{aligned}
$$

We conclude that this function can satisfy the inequality (5.4) as $t \rightarrow \infty$, that is, the inequality

$$
\begin{aligned}
\beta(t) & =\frac{1}{\tau-\delta}-\frac{1}{2 t} \frac{\tau+\delta}{\tau-\delta}-\frac{\tau-\delta}{12 t^{2}}+O\left(\frac{1}{t^{3}}\right) \\
& \leq \frac{1}{\tau-\delta}-\frac{p(\tau+\delta)}{2(\tau-\delta) t}
\end{aligned}
$$

only if $p \leq 1$.

\section{Acknowledgments}

This research was supported by the Grant no. 1/0090/09 of the Grant Agency of Slovak Republic (VEGA) and by the project APVV-0700-07 of Slovak Research and Development Agency.

\section{References}

[1] O. Arino and M. Pituk, "Convergence in asymptotically autonomous functional-differential equations," Journal of Mathematical Analysis and Applications, vol. 237, no. 1, pp. 376-392, 1999.

[2] O. Arino and M. Pituk, "More on linear differential systems with small delays," Journal of Differential Equations, vol. 170, no. 2, pp. 381-407, 2001.

[3] F. V. Atkinson and J. R. Haddock, "Criteria for asymptotic constancy of solutions of functionaldifferential equations," Journal of Mathematical Analysis and Applications, vol. 91, no. 2, pp. 410-423, 1983.

[4] H. Bereketoğlu and M. Pituk, "Asymptotic constancy for nonhomogeneous linear differential equations with unbounded delays," Discrete and Continuous Dynamical Systems. Series A, vol. 2003, supplement, pp. 100-107, 2003.

[5] J. Čermák, "Asymptotic bounds for linear difference systems," Advances in Difference Equations, vol. 2010, 14 pages, 2010.

[6] J. Čermák, "On matrix differential equations with several unbounded delays," European Journal of Applied Mathematics, vol. 17, no. 4, pp. 417-433, 2006. 
[7] J. Čermák and P. Kundrát, "Linear differential equations with unbounded delays and a forcing term," Abstract and Applied Analysis, no. 4, pp. 337-345, 2004.

[8] J. Diblík, "Asymptotic convergence criteria of solutions of delayed functional differential equations," Journal of Mathematical Analysis and Applications, vol. 274, no. 1, pp. 349-373, 2002.

[9] J. Diblík, “Asymptotic representation of solutions of equation $\dot{y}(t)=\beta(t)[y(t)-y(t-\tau(t))]$," Journal of Mathematical Analysis and Applications, vol. 217, no. 1, pp. 200-215, 1998.

[10] J. Diblík and M. Růžičková, "Exponential solutions of equation $\dot{y}(t)=\beta(t)[y(t-\delta)-y(t-\tau)]$," Journal of Mathematical Analysis and Applications, vol. 294, no. 1, pp. 273-287, 2004.

[11] J. Diblík and M. Růžičková, "Convergence of the solutions of the equation $\dot{y}(t)=\beta(t)[y(t-\delta)-y(t-\tau)]$ in the critical case," Journal of Mathematical Analysis and Applications, vol. 331, no. 2, pp. 1361-1370, 2007.

[12] B. Dorociaková and R. Olach, "Existence of positive solutions of delay differential equations," Tatra Mountains Mathematical Publications, vol. 43, pp. 63-70, 2009.

[13] I. Györi and L. Horvath, "Asymptotic constancy in linear difference equations: limit formulae and sharp conditions," Advances in Difference Equations, vol. 2010, 20 pages, 2010.

[14] I. Györi and M. Pituk, "Comparison theorems and asymptotic equilibrium for delay differential and difference equations," Dynamic Systems and Applications, vol. 5, no. 2, pp. 277-302, 1996.

[15] L. Hanuštiaková and R. Olach, "Nonoscillatory bounded solutions of neutral differential systems," Nonlinear Analysis, vol. 68, no. 7, pp. 1816-1824, 2008.

[16] T. Krisztin, "A note on the convergence of the solutions of a linear functional-differential equation," Journal of Mathematical Analysis and Applications, vol. 145, no. 1, pp. 17-25, 1990.

[17] K. Murakami, "Asymptotic constancy for systems of delay differential equations," Nonlinear Analysis, vol. 30, no. 7, pp. 4595-4606, 1997.

[18] Z. Šmarda, "Singular cauchy initial value problem for certain classes of integro-differential equations," Advances in Difference Equations, vol. 2010, Article ID 810453, 13 pages, 2010.

[19] B. Z. Vulich, Short Course of Theory of Functions of a Real Variable, An Introduction to the Integral Theory, Nauka, Moscow, Russia, 2nd edition, 1973. 